\title{
Multiscale Modeling of Thermal Conductivity of Polymer/Carbon Nanocomposites
}

\author{
T. C. Clancy, S .J. V. Frankland \\ National Institute of Aerospace \\ 100 Exploration Way \\ Hampton, VA 23666 \\ J. A. Hinkley, T. S. Gates \\ NASA Langley Research Center \\ Hampton, VA 23681
}

\begin{abstract}
:
Molecular dynamics simulation was used to estimate the interfacial thermal (Kapitza) resistance between nanoparticles and amorphous and crystalline polymer matrices. Bulk thermal conductivities of the nanocomposites were then estimated using an established effective medium approach. To study functionalization, oligomeric ethylene-vinyl alcohol copolymers were chemically bonded to a single wall carbon nanotube. The results, in a poly(ethylene-vinyl acetate) matrix, are similar to those obtained previously for grafted linear hydrocarbon chains. To study the effect of noncovalent functionalization, two types of polyethylene matrices. -- aligned (extended-chain crystalline) vs. amorphous (random coils) were modeled. Both matrices produced the same interfacial thermal resistance values. Finally, functionalization of edges and faces of platelike graphite nanoparticles was found to be only modestly effective in reducing the interfacial thermal resistance and improving the composite thermal conductivity.
\end{abstract}

\section{Introduction}

Polymers with improved thermal and electrical conductivity would have many applications [1]. Although addition of highly-conducting nanoparticles to polymer matrices is an active area of research, it is often found that thermal conductivities of the resulting nanocomposites fall well below a rule-of-mixtures-type additivity [2]. The reason for this is assumed to be a large interfacial (Kapitza) thermal resistance between the nanoparticle and the surrounding polymer matrix. Modeling has been used to explore 
trends in this resistance with particle shape, particle size [3-5] and interfacial molecular structure [6].

Carbon nanotubes (CNT) are known to have extremely high thermal conductivity [7], and research in measurement and modeling of carbon nanotube composite materials is currently very active. In a previous paper [8], functionalization of single-walled carbon nanotubes (SWCNT) by covalent grafting of alkane chains to the tube walls was studied. It was shown using molecular dynamics (MD) simulations that functionalization could reduce the interfacial thermal resistance with a vinyl polymer by an order of magnitude.

The present paper applies similar methodology to a new grafting architecture. In addition, because of the difficulty of controlling covalent functionalization and its potential for damaging the inherent properties of the CNT, possible non-covalent interactions are also explored - specifically, the effect of ordering of chains at the interface (coiled vs. extended-chain crystalline). Finally, a less-expensive filler, graphite nanoplatelets (GNP) is considered [9, 10].

The multiscale approach involves atomistic MD simulations to establish key structure-property relationships for interfacial thermal resistance. To do this, the grafting density, $\sigma$, and length, $n$, of linear hydrocarbon chains $\left(-\mathrm{C}_{n} \mathrm{H}_{2 n+1}\right)$ covalently bonded to the nanoparticle are systematically varied and, in a separate study, the matrix ordering is specified. Effects on the thermal conductivity of the composite are then predicted parametrically using an effective medium approach. Key parameters in these analytical predictions are nanoparticle aspect ratio, volume fraction, and interfacial thermal resistance. 
In Section 2, the methods for constructing the models and performing the analyses are described. In Section 3, the results from these analyses are presented and discussed. Section 4 summarizes the conclusions.

\section{Method}

The current work involves three steps. First, atomistic structures representative of the functionalized nanoparticle in the polymer matrix are generated using standard MD techniques. Next, the methodology in references [3-5] is applied to calculate the interfacial thermal resistance, $R_{K}$, in these structures. Finally, these $R_{K}$ values are used in an analytical model of the corresponding nanocomposites.

\subsection{Structure preparation}

Three sets of structures were prepared. Due to their complex architectures, care must be taken in order to produce results that are representative of the actual structure. The first set involves ethylene vinyl alcohol (EVOH) chains grafted to a single-walled carbon nanotube (SWCNT) embedded in an ethylene vinyl acetate (EVA) matrix. Each simulation cell was constructed as follows:

A $(6,6)$ SWCNT consisting of 577 carbon atoms was functionalized with 0,5 , or 10 carboxylic acid groups (corresponding to grafting densities $\sigma=0,0.00338$, or $0.006777 \AA^{-2}$ ). Ten EVOH copolymer chains, each consisting of a random sequence of 10 vinyl alcohol monomers and 15 ethylene monomers were then generated. To model the grafting chemistry, a short constant volume, constant temperature (NVT) MD simulation was run in order to allow the copolymer chains to condense around the SWCNT. Then covalent ester links were formed between vinyl alcohol groups on the copolymers and the carboxylic acid groups on the SWCNT as indicated by Eq. (1). 
Hydroxyl groups are in excess in this reaction, and all carboxylic groups react; water molecules were removed from the simulation. This functionalization architecture is different from the end-grafting of linear chains that was modeled in ref. [8].

SWNCT-COOH + HO-Copolymer $->$ SWCNT-CO-O-Copolymer $+\mathrm{H}_{2} \mathrm{O}$

The functionalized tube was embedded in an amorphous ethylene-vinyl acetate (EVA) copolymer matrix consisting of 60 chains of EVA containing 25 repeat units each. The $15 \%$ vinyl acetate monomers by weight were inserted randomly. Since the vinyl acetate mole fraction was only $5.5 \%$, each chain contained only $1-2$ vinyl acetate monomers. Each of the three grafting densities was simulated 3 times, for a total of 9 of these structures. Bulk density was effected through application of periodic boundary conditions [11]. In all the simulations, the PCFF force field [12] was used with the LAMMPS $[13,14]$ MD simulation software. The SWCNT was considered to be continuous through the $z$-axis periodic boundary coordinate; its atoms were not otherwise constrained in any way.

The bulk atomistic nanocomposite model was constructed by compression from low density. This procedure began by placing the SWCNT with its grafted chains and the polymer molecules in a box with periodic boundary conditions. Initially, the density was about $5 \%$ of the bulk density of $0.8 \mathrm{~g} / \mathrm{cm}^{3}$. To prevent the polymer chains from collapsing, the nonbond pair potential used to describe the forces between atoms which are not chemically bonded to each other was scaled by a factor of 0.001 and a short cutoff (3.0 ̊) was applied with electrostatic interactions between the partial charges on each 
atom turned off. To allow the polymer chains to diffuse through the periodic box and achieve random configurations, the MD simulations were run for $50 \mathrm{ps}$ using a $1 \mathrm{fs}$ timestep at $500 \mathrm{~K}$. The box volume was reduced linearly with time to give a density about $10 \%$ of the final bulk density. A second MD run condensed the system to a density of about $50 \%$ of the final bulk density over 50 ps at $500 \mathrm{~K}$. During this run, the unscaled nonbond pair potentials were then applied with a cutoff of $9.0 \AA$, and electrostatic interactions between the partial charges on each atom were included via the Ewald summation technique. At the end of this run, energy minimization was performed on the system, followed by constant pressure MD simulation at $300 \mathrm{~K}$ and 1 atm of pressure. After that, MD was applied for $10 \mathrm{ps}$ while increasing the pressure to $100 \mathrm{~atm}$. This was followed by 50 ps of MD simulation. The pressure was reduced from 100 to 1 atm over $10 \mathrm{ps}$, followed by a MD run at 1 atm and $300 \mathrm{~K}$ for 100 ps. Such equilibration procedures are typical for the simulation of polymers[15, 16]. Running MD at high temperatures usually allows the polymers to relax out of any nonphysical configurations. Running MD with elevated pressures helps to achieve an appropriate condensed phase density.

The second set of structures involves a SWCNT surrounded by two different polyethylene (PE) matrices. The first of these, amorphous polyethylene, was constructed in a manner identical to the one described above but using 78 chains of 45 carbon atoms $\left(\mathrm{C}_{45} \mathrm{H}_{92}\right)$ as the matrix. A second model was constructed in which the polyethylene chains were aligned parallel with the SWCNT axis. The chain length (45 carbon atoms, $\mathrm{C}_{45} \mathrm{H}_{92}$ ) was chosen to fit in the space available, with the SWCNT extended through the $z$-axis periodic boundary conditions. The $78 \mathrm{C}_{45} \mathrm{H}_{92}$ chains were arranged parallel with an 
initial density of about $70 \%$ of the final equilibrium density. Energy minimization with a convergence criterion of $10 \mathrm{kcal} / \mathrm{mol}$ was first applied to the configuration. MD was applied for $10 \mathrm{ps}$ while increasing the pressure from 1 to 100 atm at $300 \mathrm{~K}$. This was followed by a compression at $100 \mathrm{~atm}$ for $50 \mathrm{ps}$ at $300 \mathrm{~K}$. The pressure was reduced from 100 to $1 \mathrm{~atm}$ over $10 \mathrm{ps}$, followed by an MD run at $1 \mathrm{~atm}$ for $400 \mathrm{ps}$ and $300 \mathrm{~K}$. Fig. 1 shows axial views of these two polyethylene-SWCNT atomistic models. The ordered matrix shows the polymer chains retaining their orientation parallel to the axis of the nanotube after the equilibration run.

The third set of structures involves the GNP in an EVA matrix. Because the planar dimensions of a GNP are still very large compared to the typical volume of an atomistic MD simulation, the platelets are simulated as (infinite) sheets extended through the periodic boundary conditions. Two surfaces (basal plane and edge) are simulated separately. To model the flat surface, 3 sheets of graphite each containing 836 carbon atoms were oriented in the $x z$ plane, with periodic boundary conditions along the $x$ and $z$ directions. The normal to the graphite plane is the $y$ coordinate as shown in Fig. 2. To model the edge of the graphite particle, 13 sheets of graphite, each containing 228 carbon atoms, were oriented in the $x z$ plane with periodic boundary conditions along the $x$ and $y$ directions as shown in Fig. 3.

Surfaces and edges are functionalized by forming covalent chemical bonds with short linear hydrocarbon chains $\left(\right.$ GNP- $\left.\mathrm{C}_{n} \mathrm{H}_{2 n+1}\right)$ with a range of grafting densities. The GNP/EVA nanocomposite models are prepared via the same methodology used for the SWCNT nanocomposites, with the exception that the graphite atoms are held fixed during the condensation procedure. The final model uses periodic boundary conditions in 
the $x y z$ directions. The polymer is sandwiched between the graphite structure in a condensed phase.

\subsection{Simulation of the heat transfer}

Following the last MD run of the atomistic structures at $300 \mathrm{~K}$ described in Section $2.1, R_{K}$, the interfacial thermal resistance was calculated [3-5] by instantaneously heating the nanoparticle atoms to a temperature of $500 \mathrm{~K}$ and then monitoring the difference in temperature, $\Delta T$, between the nanoparticle atoms and the matrix atoms during a constant energy simulation (NVE ensemble). A plot of $\ln (\Delta T) v s$. time yields a slope which is the negative inverse of the characteristic decay time, $\tau$. Eq. (2) then gives the interfacial thermal resistance, $R_{K}$, where $c_{T} / A_{T}$ is the heat capacity per area of nanoparticle surface. This ratio $\left(c_{T} / A_{T}\right)$ is calculated assuming a value of $0.71 \mathrm{~J} / \mathrm{gK}$ for $c_{T}$ [17]. This results in a value of $5.6 \times 10^{-4} \mathrm{~J} / \mathrm{m}^{2} \mathrm{~K}$ for $c_{T} / A_{T}$ for both the SWCNT and the flat surface of the GNP while a value of $3.1 \times 10^{-4}$ is obtained for the edge surface of the GNP.

$$
R_{K}=\frac{\tau}{\left(c_{T} / A_{T}\right)}
$$

\subsection{Analytical modeling of the thermal conductivity}

As was done previously for functionalized SWCNT nanocomposites [8], thermal conductivities of nanocomposites were calculated using the interfacial resistance values from the heat transfer simulations. For a dilute dispersion of randomly oriented prolate ellipsoidal inclusions (dimensions $a_{1}=a_{2}>a_{3}$ ), an effective medium[18, 19] approach yields Eq. (3)

$$
K_{e}=K_{m} \frac{3+f\left[2 \beta_{11}\left(1-L_{11}\right)+\beta_{33}\left(1-L_{33}\right)\right]}{3-f\left[2 \beta_{11} L_{11}+\beta_{33} L_{33}\right]}
$$


where $K_{e}$ is the thermal conductivity of the composite, $K_{m}$ is the thermal conductivity of the matrix and $f$ is the volume fraction of the inclusion. The indices ' 1 ' and ' 3 ' refer to the dimensions $a_{1}$ and $a_{3}$ respectively. Fig. 4 depicts this ellipsoid. The subscripts (ii) in $L_{i i}$ refer to the coordinates of the GNP, where ' 3 ' is the direction perpendicular to the face of the ellipsoid shaped GNP particle and $\beta_{i i}$ is defined as:

$$
\beta_{i i}=\frac{K_{i i}^{c}-K_{m}}{K_{m}+L_{i i}\left(K_{i i}^{c}-K_{m}\right)}
$$

and $L_{i i}$ is defined as:

$$
\begin{gathered}
L_{11}=L_{22}=\frac{p^{2}}{2\left(p^{2}-1\right)}+\frac{p}{2\left(1-p^{2}\right)^{3 / 2}} \cos ^{-1} p, \\
L_{33}=1-2 L_{11}
\end{gathered}
$$

respectively, where $p=a_{3} / a_{1}$ is the aspect ratio of the ellipsoid. The values $K_{i i}^{c}$, are defined as

$$
\begin{gathered}
K_{11}^{c}=\frac{K_{c_{\text {parallel }}}}{1+\frac{2 R_{K_{\text {parallel }}} K_{c_{\text {parallel }}}}{a_{1}}} \\
K_{33}^{c}=\frac{K_{c_{\text {perpendicular }}}}{1+\frac{2 R_{K_{\text {perpendicular }}} K_{c_{\text {perpendicular }}}}{a_{3}}}
\end{gathered}
$$

where $K_{c_{\text {perpendicular }}}$ and $K_{c_{\text {parallel }}}$ are the thermal conductivities of the graphite layer in the perpendicular and parallel directions respectively. In evaluating Eqs. (2)-(8), we used $K_{m}=0.2 \mathrm{~W} / \mathrm{mK}, K_{c_{\text {parallel }}}=390 \mathrm{~W} / \mathrm{mK}$ [17], and $K_{c_{\text {perpendicular }}}=2 \mathrm{~W} / \mathrm{mK}$ which are the values given for pyrolytic graphite. This should be a reasonable estimation for the GNP 
properties. The interfacial thermal resistance values $R_{K_{\text {perpendicular }}}$ and $R_{K_{\text {parallel }}}$ are those calculated from atomistic MD simulations.

\section{Results and Discussion}

The following three subsections describe results for the EVOH-functionalized nanotube, the functionalized nanotube in ordered and amorphous polyethylene matrices, and GNP nanocomposite modeling, respectively.

\subsection{EVOH functionalized nanotube/EVA}

The nanocomposite model was described in Section 2.1. Fig. 5 shows the interfacial thermal resistance, $R_{K}$, as a function of grafting density, $\sigma$. Error bars shown are standard deviations among runs for all 3 independent configurations. Although the effect of ester-linked EVA on $R_{K}$ is substantial, it is noticeably less than the effect seen with directly grafted linear hydrocarbon chains [8]. Thus as might have been expected, the coupling of SWCNT vibrations to the polymeric matrix [5] is seen to be sensitive to the chemical bond topology adjacent to the tube. These results also serve to illustrate the value of MD simulation in providing guidance on chemical functionalization with the greatest potential payoff.

\subsection{SWCNT/Amorphous and Ordered Polyethylene (PE)}

Two different types of PE/CNT composites were constructed. In one type, the matrix chains were amorphous random coils; in the other, the PE chains were oriented parallel with the axis of the carbon nanotube. Fig. 1 shows views along the $z$-axis of the amorphous and ordered matrix atom configurations. As before, there were 5 thermal MD runs for each of 3 independent configurations. The values obtained for the two matrix types are identical $\left(10.9 \pm 0.63 \times 10^{-8} \mathrm{~m}^{2} \mathrm{~K} / \mathrm{W}\right.$ for the amorphous PE and $10.9 \pm 1.9 \times 10^{-8}$ 
$\mathrm{m}^{2} \mathrm{~K} / \mathrm{W}$ for the oriented PE). They are also consistent with the results in Fig. 5 for the unfunctionalized tube for $\sigma=0$. To examine this further, the radial distribution of the mass density, $\rho$, is shown in Fig. 6 for the three matrix types: the amorphous PE, the ordered PE and the EVA, each surrounding an unfunctionalized SWCNT. There is little distinction between the amorphous PE and the EVA. This is as expected, since there is relatively little vinyl acetate content in the EVA. The ordered PE simulation is clearly distinct from the other two, however. Thus, it is seen that modest changes in the matrix have little effect on the interfacial thermal resistance, at least for small $(6,6)$ SWCNTs. Covalent functionalization of the tube, on the other hand, gave qualitatively different results, presumably by changing phonon coupling [3].

\subsection{Functionalized Graphite Nanoplatelet (GNP) Composites}

Fig. 7 shows calculated interfacial thermal resistances for both graphite surfaces as a function of the grafting density. One interesting feature is that the $R_{K}$ values for the edge are substantially higher than for the flat graphite sheet surface, perhaps because the in-plane thermal motions cannot couple efficiently to the matrix. In contrast to results for a functionalized SWCNT [8] the length of the grafted chain appears to have no influence within the range studied here $(n=8-18)$. The net effect of increasing grafting density is also much smaller for the graphite surfaces than for the SWCNT.

The interfacial thermal resistance values calculated with the MD simulations can be used with the effective medium equations of Section 2.3 to predict the effect of the functionalization on the thermal conductivity of the nanocomposite. The particles are modeled as oblate ellipsoids with dimensions $a_{3}=1 \times 10^{-8} \mathrm{~m}$ and $a_{1}=5 \times 10^{-6} \mathrm{~m}$. Considering first the functionalization of the larger flat surfaces of the GNP, Fig. 8 shows 
thermal conductivity of the composite as a function of $R_{K_{\text {perpendicular }}}$, calculated using Eqs. (2)-(8). Even with $R_{K_{\text {perpendicular }}}$ values approaching zero, the predicted thermal conductivity of the composite is only about 3 times that of the matrix at the highest loading of GNP (5\% volume).

Considering next the effect of functionalizing the edges of the GNP, Fig. 9 shows predicted composite thermal conductivities as a function of $R_{K_{\text {parallel }}}$. In this case, increases in thermal conductivity of over a factor of 15 are predicted at low values of $R_{K_{\text {parallel }}}$. The stronger dependence of thermal conductivity on $R_{K_{\text {parallel }}}$ versus $R_{K_{\text {perpendicular }}}$ is due to the greater conductivity of graphite along the parallel direction versus the perpendicular direction. The low $R_{K_{\text {parallel }}}$ values in Fig 9. which would predict significant thermal conductivity increases are below those calculated by the MD simulation (indicated by the symbols) and may not be accessible experimentally. Thus, although functionalization of the faces and edges of GNPs can be expected to reduce interfacial resistances by $1 / 3$ to $1 / 2$, the effect on the thermal conductivity of the composite is not large. This result contrasts with the conductivity predictions for carbon nanotube composites in [8], which easily rose an order of magnitude above the base polymer. Both the thermal conductivity of the particle itself and the assumed aspect ratio were higher for the CNT.

If the results for 0 and 20 weight $\%$ loading in reference [10] are interpolated to 12 weight $\%$ ( = 5 volume $\%$ ), the thermal conductivity obtained is $0.53 \mathrm{~W} / \mathrm{mK}$. This is in somewhat close agreement to the value plotted in Fig. 8 for 5 volume $\%$ and no functionalization $(0.6 \mathrm{~W} / \mathrm{mK})$. Experimentally, the effectiveness of plate-like GNPs in 
improving thermal conductivity varies considerably $[10,20,21]$. The analytical model is useful in exploring various parameters in addition to functionalization. For example, comparison of Figs. 9 and 10 shows the effect of changing the assumed aspect ratio of the ellipsoidal particle from 500 to 5000. All other values were unchanged. Although the composite thermal conductivities are substantially higher in Fig. 10, the overall improvement in thermal conductivity over the neat matrix is still modest.

\section{Conclusions}

Based on comparisons of amorphous and crystalline matrices, there does not appear to be any evidence that a "noncovalent functionalization" approach in the interfacial region will play a major role in reducing the interfacial thermal resistance and thereby increasing the thermal conductivity of the composite.

MD results from EVOH functionalization of the SWCNT indicate that a variety of functionalization architectures are useful in achieving lower thermal interfacial resistance and therefore higher thermal conductivity.

Simulations of GNP particles in EVA clearly predict that alkane functionalization should not result in an increase in thermal conductivity comparable to that seen with SWCNT filler. This is due to three factors: lower assumed conductivities of the GNP; the slightly higher interfacial resistance of the GNP $\left(15-30 \times 10^{-8} \mathrm{~m}^{2} \mathrm{~K} / \mathrm{W}\right)$; and a smaller assumed aspect ratio. The multiscale approach presented here may be useful in optimizing nanocomposite properties. 


\section{References:}

[1] King JA, Weber EH, Quan CL, Tucker KW, Vogt BD. Journal of Composite Materials 2000;34:2038-2060.

[2] Biercuk MJ, Llaguno MC, Radosavljevic M, Hyun JK, Johnson AT, Fischer JE. Applied Physics Letters 2002;80:2767-2769.

[3] Huxtable ST, Cahill DG, Shenogin S, Xue LP, Ozisik R, Barone P, Usrey M, Strano MS, Siddons G, Shim M, Keblinski P. Nature Materials 2003;2:731-734.

[4] Shenogin S, Bodapati A, Xue L, Ozisik R, Keblinski P. Applied Physics Letters 2004;85:2229-2231.

[5] Shenogin S, Xue LP, Ozisik R, Keblinski P, Cahill DG. Journal of Applied Physics 2004;95:8136-8144.

[6] Patel HA, Garde S, Keblinski P. Nano Letters 2005;5:2225-2231.

[7] Berber S, Kwon YK, Tomanek D. Physical Review Letters 2000;84:4613-4616.

[8] Clancy TC, Gates TS. Polymer 2006;47:5990-5996.

[9] Chasiotis I, Chen Q, Odegard GM, Gates TS. Experimental Mechanics 2005;45:507-516.

[10] Ghose S, Watson KA, Working DC, Connell JW, Smith JG, Sun YP. Composites Science and Technology 2008;68:1843-1853.

[11] Allen MP, Tildesley DJ. Computer Simulation of Liquids. Oxford: Oxford University Press, 1987.

[12] Hwang MJ, Stockfisch TP, Hagler AT. Journal of the American Chemical Society 1994;116:2515-2525.

[13] http://www.cs.sandia.gov/ sjplimp/lammps.html.

[14] Plimpton S. Journal of Computational Physics 1995;117:1-19.

[15] Goudeau S, Charlot M, Muller-Plathe F. Journal of Physical Chemistry B 2004;108:18779-18788.

[16] Annis BK, Borodin O, Smith GD, Benmore CJ, Soper AK, Londono JD. Journal of Chemical Physics 2001;115:10998-11003.

[17] Pierson HO. Handbook of Carbon, Graphite, Diamond and Fullerenes: Properties, Processing and Applications (Materials Science and Process Technology): Noyes Publications, 1994.

[18] Nan CW, Liu G, Lin YH, Li M. Applied Physics Letters 2004;85:3549-3551.

[19] Nan CW, Birringer R, Clarke DR, Gleiter H. Journal of Applied Physics 1997;81:6692-6699.

[20] Fukushima H, T Drzal L, Rook BP, Rich MJ. Journal of Thermal Analysis and Calorimetry 2006;85:235-238.

[21] George JJ, Bhowmick AK. Journal of Materials Science 2008;43:702-708. 


\section{Amorphous Matrix}

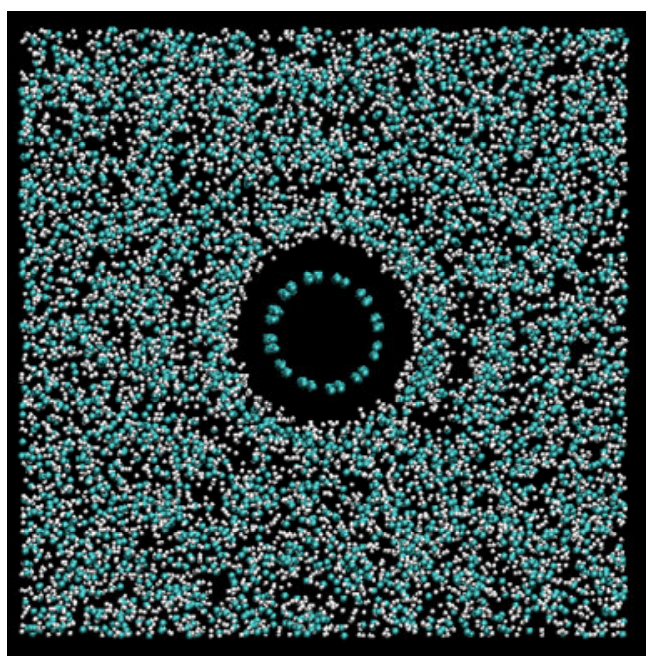

\section{Ordered Matrix}

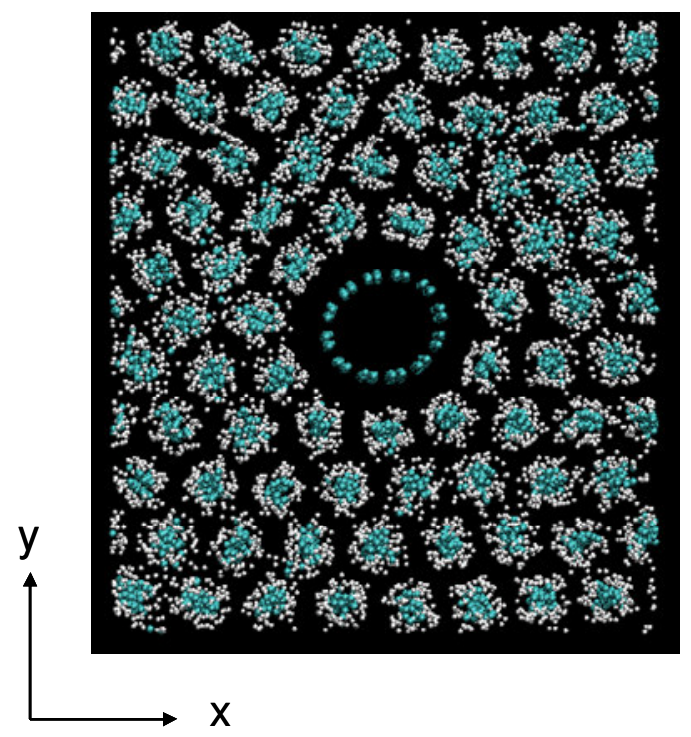

Fig. 1. Views along the $z$-axis of atomistic models of the two polyethylene matrices surrounding a single wall carbon nanotube. The periodic boxes are $4.4 \mathrm{~nm}$ in the $x$ and $y$ dimensions and $5.7 \mathrm{~nm}$ in the (nanotube axial) $z$-axis direction. 


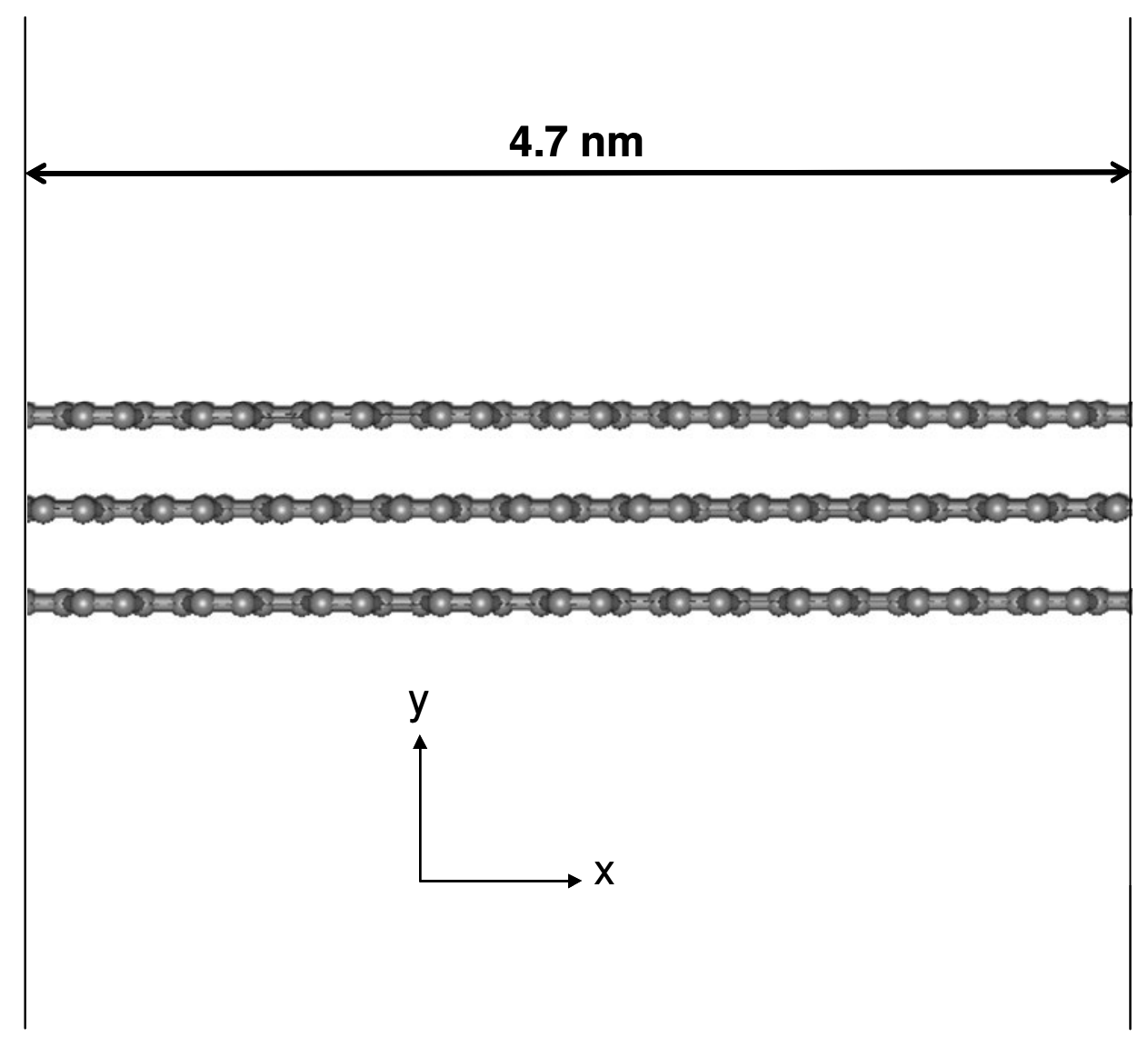

Fig. 2. Modeling the flat graphite surface using periodic boundary conditions. The periodic boundary conditions are enacted across the $x$ and $z$ coordinates. The normal to the exposed surface is along the $y$ axis. The periodic boundary dimensions are $4.7 \mathrm{~nm}$ in the $x$ and $z$ directions. Only the graphite atoms are shown in this depiction for clarity. The atoms associated with the functionalization and the matrix are not shown. 


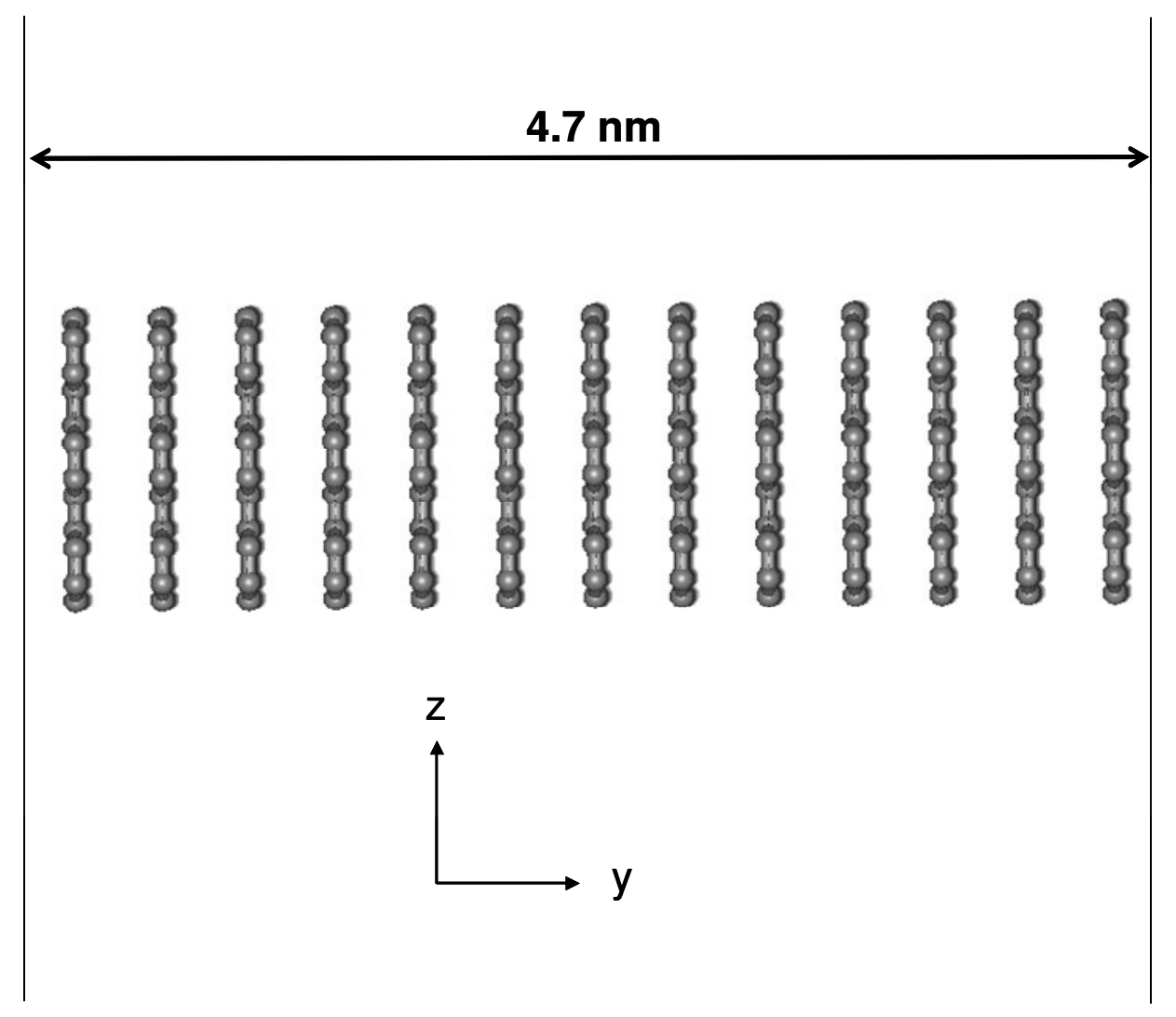

Fig. 3. Modeling the edge of the graphite particle using periodic boundary conditions. The periodic boundary conditions are enacted across the $x$ and $y$ coordinates. The normal to the exposed surface is along the $z$ axis. The periodic boundary dimensions are $4.7 \mathrm{~nm}$ in the $x$ and $y$ directions. Only the graphite atoms are shown in this depiction for clarity. The atoms associated with the functionalization and the matrix are not shown. 


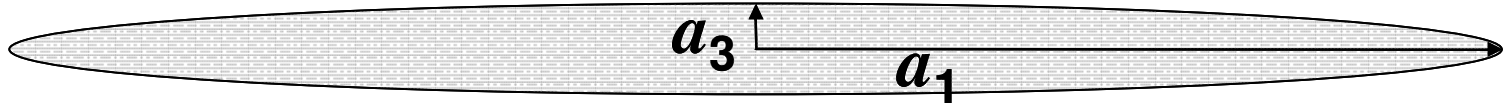

Fig. 4. The ellipsoid, depicted in two dimensions. The third dimension, $a_{2}=a_{1}$. 


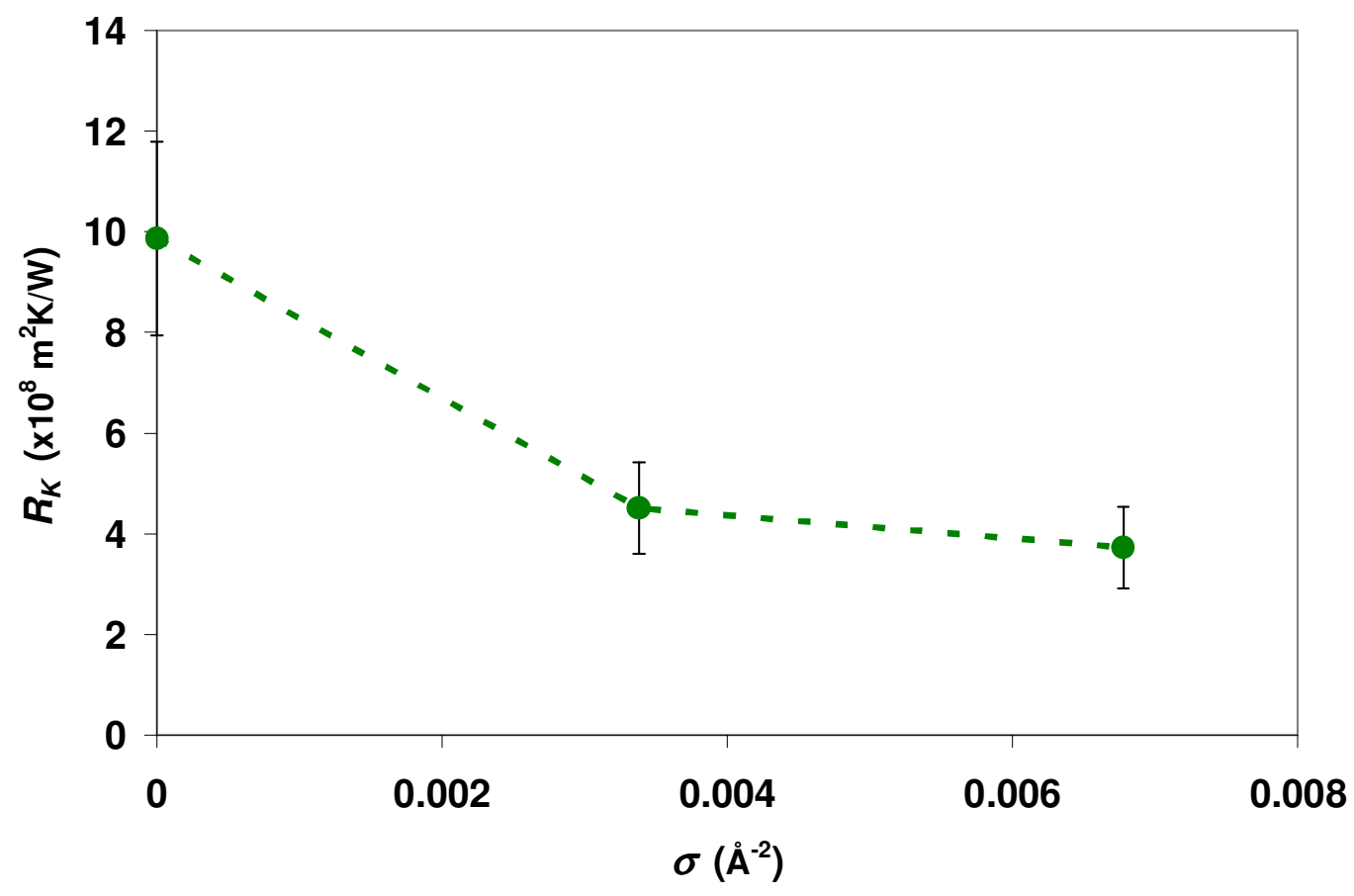

Fig. 5. The interfacial thermal resistance, $R_{K}$, as a function of the grafting density, $\sigma$, of covalent chemical bonds attached to the wall of the carbon nanotube per unit area. 


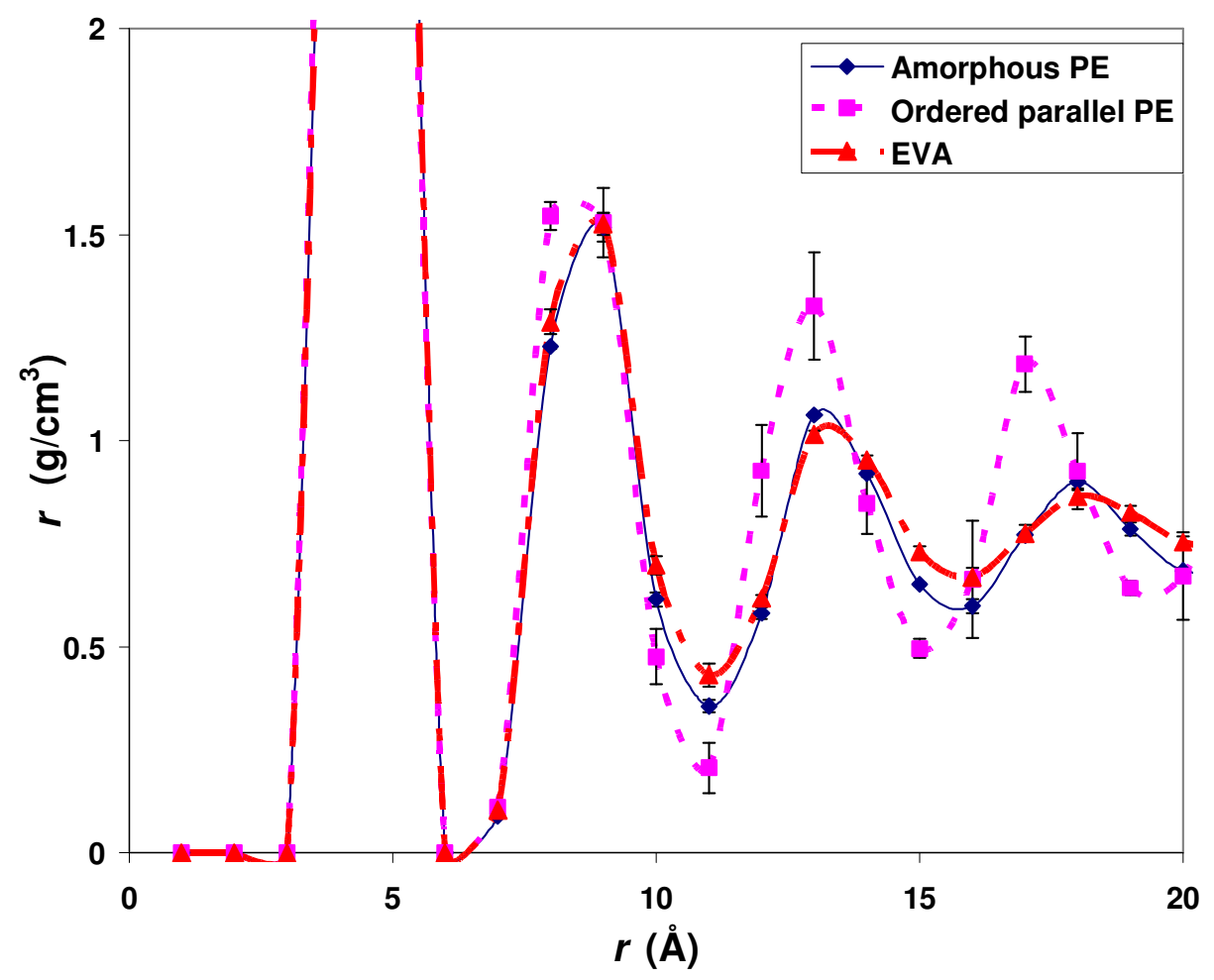

Fig. 6. The radial distribution of mass density, $\rho$, as a function of distance, $r$, from the center of the nanotube in atomistic nancomposite simulations. 


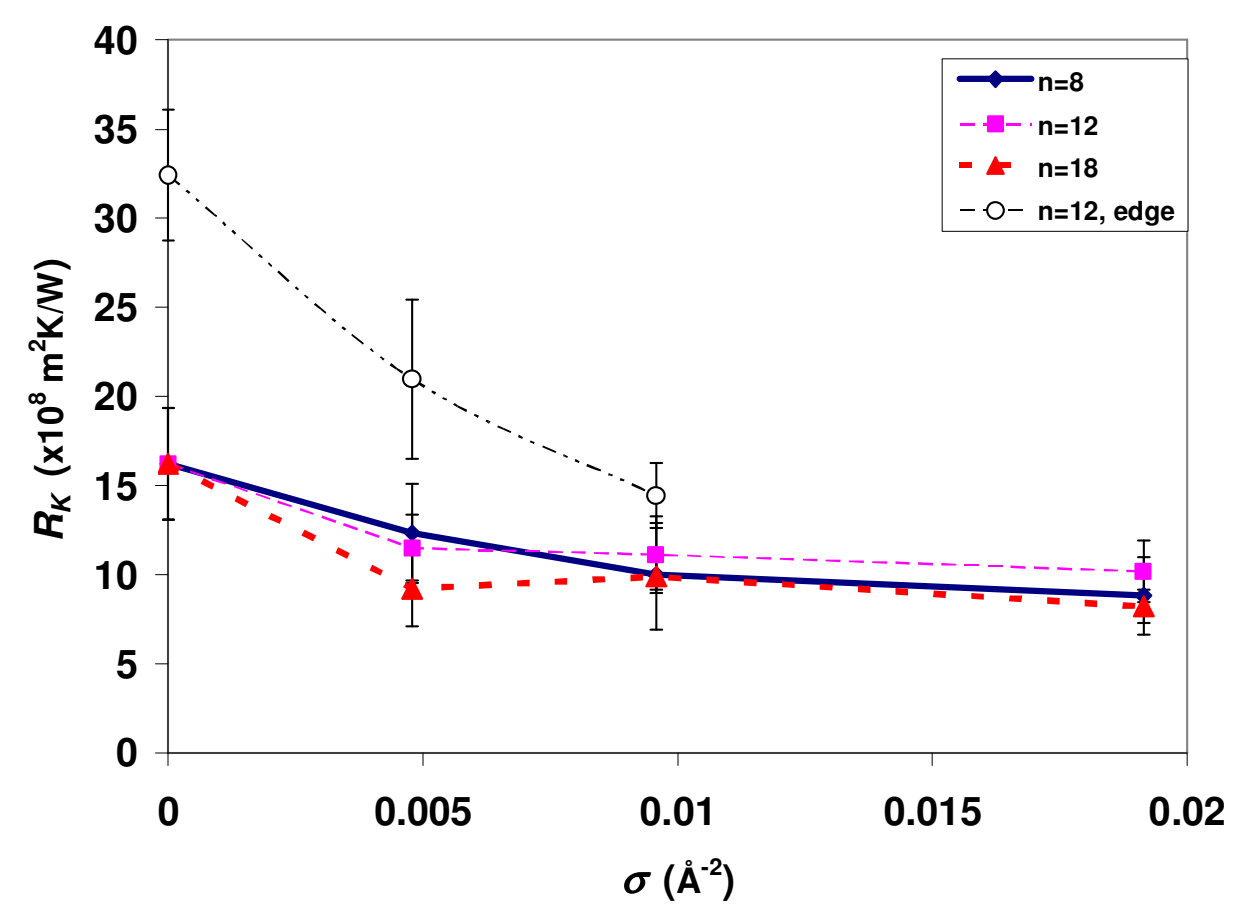

Fig. 7. The interfacial thermal resistance as a function of the grafting density, $\sigma$, of covalent chemical bonds attached to the graphite nanoparticle. The open circles indicate values calculated for the edge surface of the parallel graphite sheets with a hydrocarbon chain of length $n=12\left(\right.$ GNP- $\left.\mathrm{C}_{12} \mathrm{H}_{25}\right)$ covalently bonded. The filled diamond, square and triangle indicate values calculated for the flat surface of the graphite sheets with a hydrocarbon chain of length $n=8,12$ and $18\left(\mathrm{GNP}_{-} \mathrm{C}_{n} \mathrm{H}_{2 n+1}\right)$ covalently bonded, respectively. 


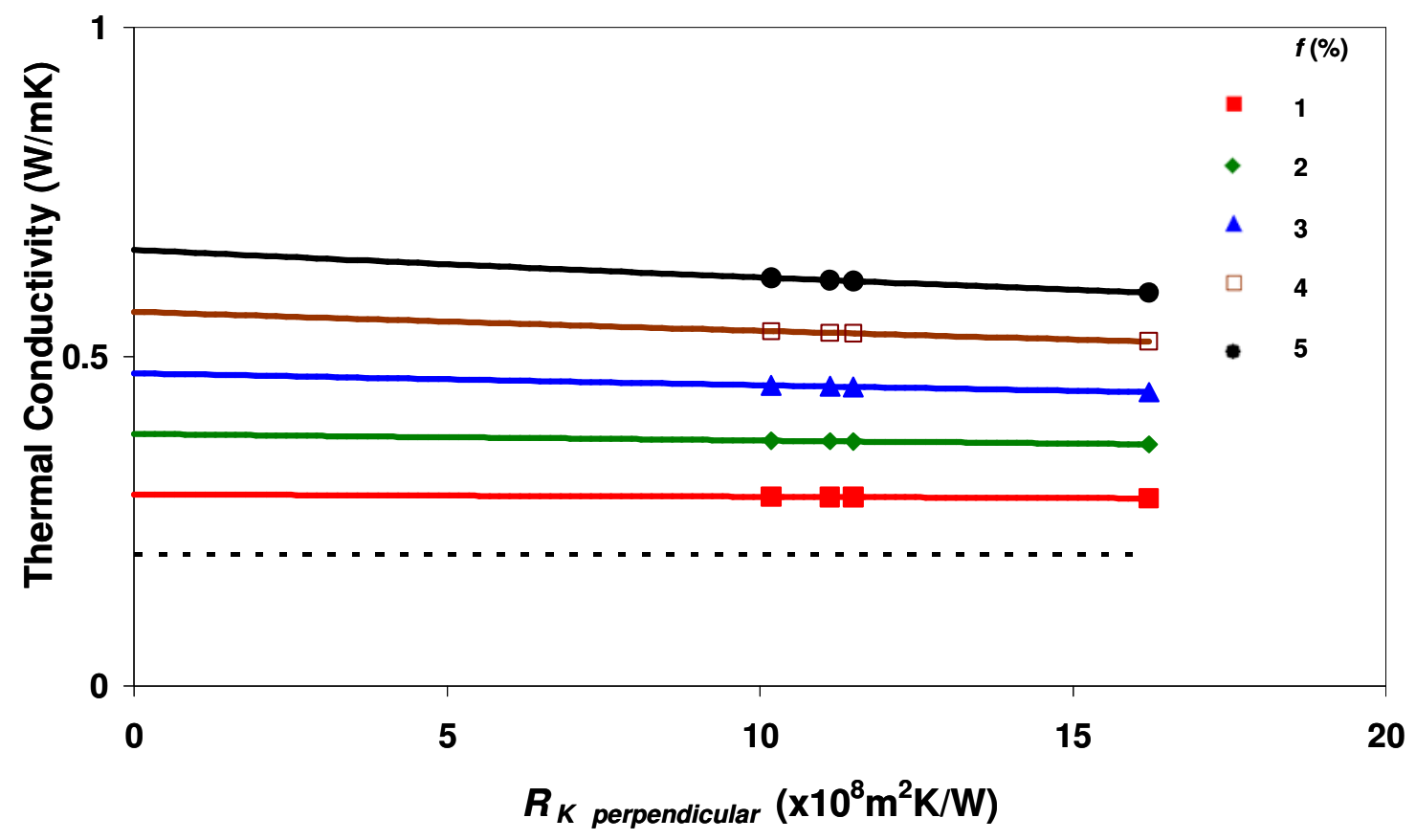

Fig. 8. The thermal conductivity of the GNP-polymer nanocomposite as a function of $R_{K_{\text {perpenticular }}}$ for varying volume fraction, $f$. The dimensions of the GNP in this example are assumed to be $a_{3}=1 \times 10^{-8} \mathrm{~m}$ and $a_{1}=5 \times 10^{-6} \mathrm{~m}$. The dotted line indicates the value for the neat matrix $(0.2 \mathrm{~W} / \mathrm{mK}$.) 


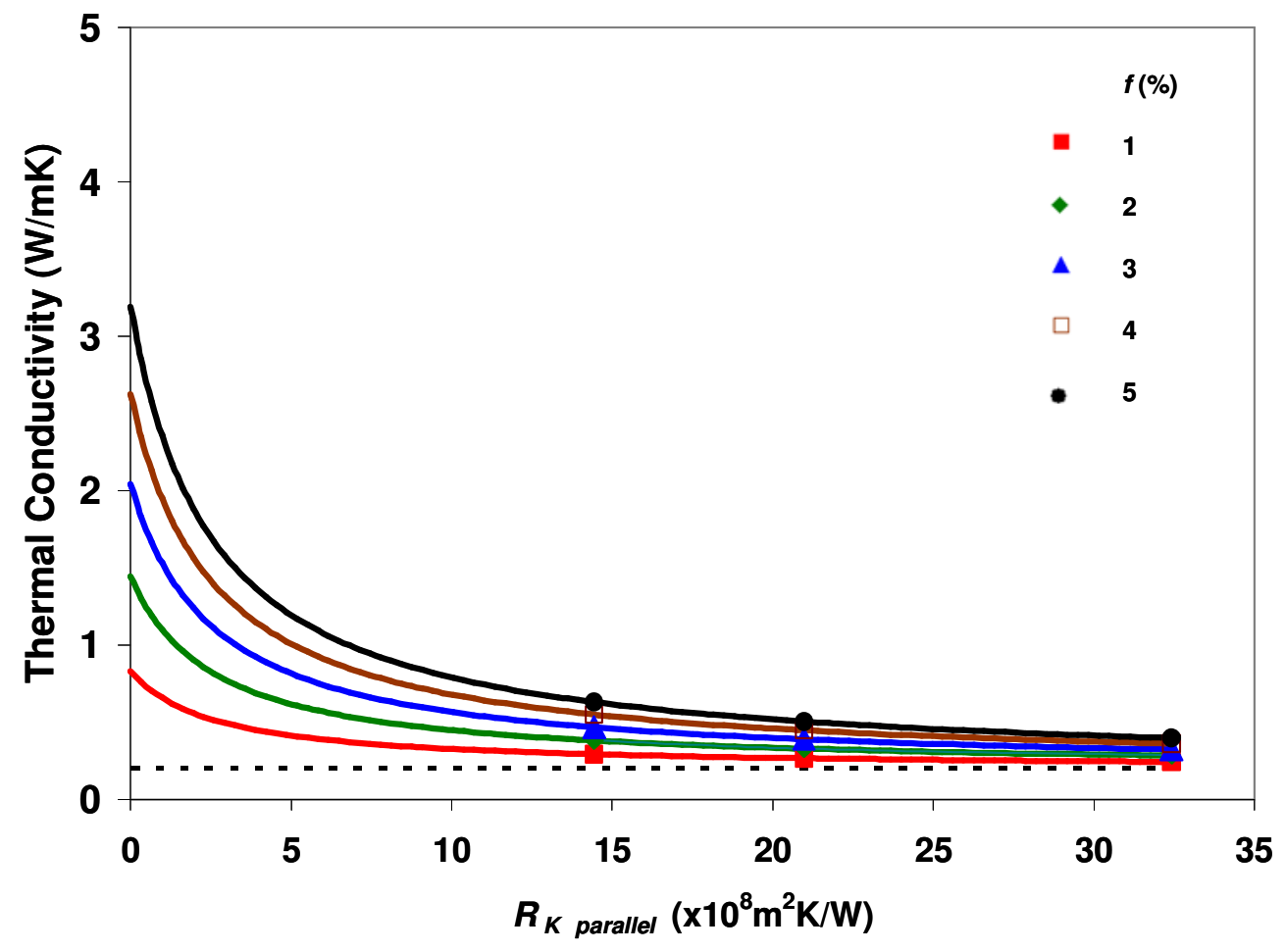

Fig. 9. The thermal conductivity of the GNP-polymer nanocomposite as a function of $R_{K_{\text {parallel }}}$ for varying volume fraction, $f$. The dimensions of the GNP in this example are assumed to be $a_{3}=1 \times 10^{-8} \mathrm{~m}$ and $a_{1}=5 \times 10^{-6} \mathrm{~m}$. The dotted line indicates the value for the neat matrix $(0.2 \mathrm{~W} / \mathrm{mK}$.) 


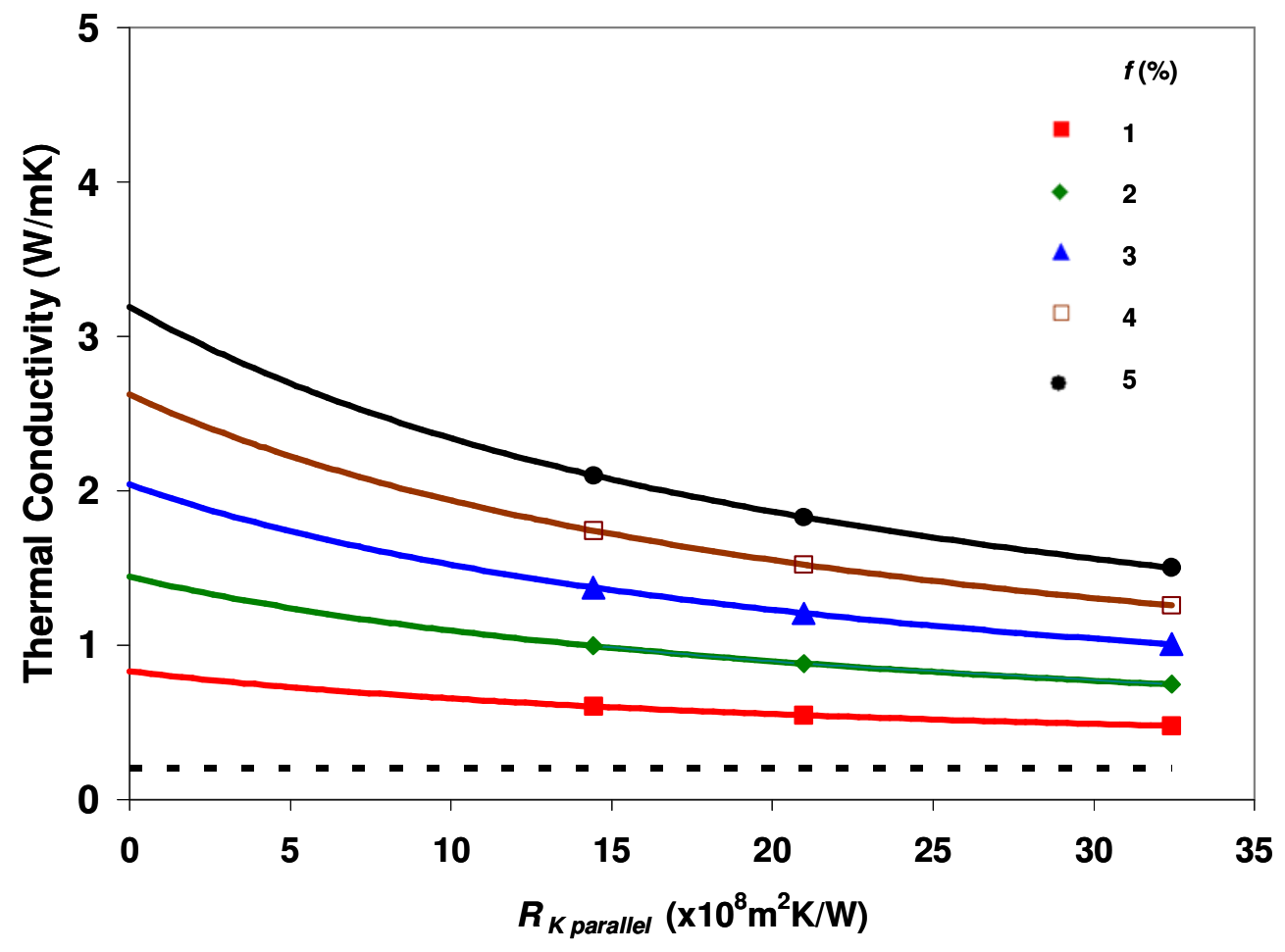

Fig. 10. The thermal conductivity of the GNP-polymer nanocomposite as a function of $R_{K_{\text {parallel }}}$ for varying volume fraction, $f$. The dimensions of the GNP in this example are assumed to be $a_{3}=1 \times 10^{-8} \mathrm{~m}$ and $a_{1}=5 \times 10^{-5} \mathrm{~m}$. The dotted line indicates the value for the neat matrix $(0.2 \mathrm{~W} / \mathrm{mK}$.) 